\title{
Forecasting the action of CAR-T cells against SARS-corona virus-II infection with branching process
}

\author{
Khaled A. Al-Utaibi ${ }^{1} \cdot$ Alessandro Nutini $^{2} \cdot$ Ayesha Sohail $^{3} \cdot$ Robia Arif $^{3} \cdot$ Sümeyye Tunc $^{4} \cdot$ Sadiq M. Sait $^{5}$
}

Received: 11 June 2021 / Accepted: 25 September 2021 / Published online: 15 October 2021

(c) The Author(s), under exclusive licence to Springer Nature Switzerland AG 2021

\begin{abstract}
The CAR-T cells are the genetically engineered T cells, designed to work specifically for the virus antigens (or other antigens, such as tumour specific antigens). The CAR-T cells work as the living drug and thus provides an adoptive immunotherapy strategy. The novel corona virus treatment and control designs are still under clinical trials. One of such techniques is the injection of CAR-T cells to fight against the COVID-19 infection. In this manuscript, the hypothesis is based on the CAR-T cells, that are suitably engineered towards SARS-2 viral antigen, by the $\mathrm{N}$ protein. The $\mathrm{N}$ protein binds to the SARS-2 viral RNA and is found in abundance in this virus, thus for the engineered cell research, this protein sequence is chosen as a potential target. The use of the sub-population of T-reg cells is also outlined. Mathematical modeling of such complex line of action can help to understand the dynamics. The modeling approach is inspired from the probabilistic rules, including the branching process, the Moran process and kinetic models. The Moran processes are well recognized in the fields of artificial intelligence and data science. The model depicts the infectious axis "virus-CAR-T cells-memory cells". The theoretical analysis provides a positive therapeutic action; the delay in viral production may have a significant impact on the early stages of infection. Although it is necessary to carefully evaluate the possible side effects of therapy. This work introduces the possibility of hypothesizing an antiviral use by CAR-T cells.
\end{abstract}

Keywords CAR-T $\cdot \mathrm{SARS}-\mathrm{CoV} 2 \cdot$ Mathematical model $\cdot$ Reproductive number $\cdot$ Moran process

Mathematics Subject Classification $15 \mathrm{~A} 18 \cdot 42 \mathrm{~A} 15 \cdot 49 \mathrm{M} 37 \cdot 65 \mathrm{~K} 05$

Alessandro Nutini

nutini@centrostudiattivitamotorie.it

Khaled A. Al-Utaibi

alutaibi@uoh.edu.sa

1 Computer Science and Software Engineering Department, University of Ha'il, Ha'il, Saudi Arabia

2 Centro Studi Attività Motorie, Department of Biology and Biomechanics, 94 via di Tiglio, loc. Arancio, 55100 Lucca, Italy

3 Department of Mathematics, Comsats University Islamabad, Lahore 54000, Pakistan

4 Physiotherapy Programme, Vocational School of Sciences, Medipol University, Unkapanı, Atatürk Bulvarı, No: 27, Halic Campus, Fatih, 34083 Istanbul, Turkey

5 Center for Communications and IT Research, Research Institute, King Fahd University of Petroleum \& Minerals, Dhahran 31261, Saudi Arabia

\section{Introduction}

In the field of computational biology, tremendous progress is achieved due to the novel and cutting edge discoveries. CAR-T cells are a promising technology that reprograms $\mathrm{T}$ cells to overcome the barriers that cancer presents against the action of the immune system: about $90 \%$ of individuals treated with CAR (Chimeric Antigen Receptor) technology who selectively target tumors expressing CD-19 such as pediatric acute lymphoblastic lymphoma, go into remission. Since the CAR receptor allows a reorganization of antigen presentation without requiring the intervention of the molecules of the Major Histocompatibility Complex (MHC), the targets that they can detect are fewer than the $\mathrm{T}$ cell receptors (TCR), but they have a rather significant advantage given by a greater specificity that eludes the escape mechanisms that pathogens or the tumor itself can implement.

A study by Roberts et al. (1994) showed the realization of a construct that directed the specificity of $\mathrm{T}$ cells against 
cells infected with the HIV (fusion of CD4 with the CD3 polypeptide chain called quot;CD4CARquot;), exploiting the particular interaction between molecules of the viral envelope with CD4. This experience, however, despite being quite safe, did not allow the observation of a lasting control of the infection in question (Scholler et al. 2012). This approach, however, has focused on the central action of $\mathrm{T}$ cells in the control of viral infections and, thanks to advances in scientific research and biotechnology, numerous advances in the control of chronic HIV inflammation, and the consideration of a subpopulation of $T$ cells that can be engineered, considering their advantages and disadvantages (Cheng et al. 2016). Thanks to positive experimentation of CAR-T cells in vivo against cancer, advances in the construction technology of the CAR receptor have led to the use of the second generation of this CD4-based receptor optimized by a 4-1BB-CD3 signal domain which was found to be much more efficient in controlling HIV viral replication in vitro and experiments on mouse showed a strong response to the infection (Leibman et al. 2017).

The second generation of CAR-T cells, thus engineered, contains a portion that binds the target antigen, that is the single-chain variable fragment ( $\mathrm{scFV}$ ), shows a good antiviral capacity in vitro, but in humans, several factors limit its use and a possible therapy need for a better effectiveness against the diversity of the biochemical characteristics of the HIV and not being immunogenic. Our group of analysis and research of mathematical models in Biology speculated if similar technology could also be applied in the case of the SARS-CoV2 pandemic that we are currently experiencing, directing a specificity of target similar to what happens for the HIV and where the action of the engineered cells themselves is somewhat simplified by the fact that, at the moment and contrary to HIV, there is no evidence that this type of coronavirus infects $\mathrm{T}$ cells. that of using Treg cells, typical of the autoimmunity process, which can proliferate in an antigen-specific way and lead to a sort of subsequent protection for the pathology that expresses that same antigen (Elinav et al. 2008).

Viral and opportunistic infections are a serious threat to the health of infected individuals and immunocompromised patients. Despite the availability of antiviral drugs, the mortality rate is still significant in high-risk patients. Therefore, therapies that provide long-term control or that are capable of eradicating the viral reservoir are needed. Viral pathogen-specific effector CD8 + T cells play a crucial role in controlling infections in immunocompetent individuals, making adoptive $\mathrm{T}$ cell therapy an attractive alternative to currently used anti-infectious therapies. $\mathrm{T}$ cells specific for viral pathogens occur at low frequencies in the patient39;s blood, making them difficult to isolate and expand. Furthermore, these cells have phenotypes subject to depletion and can be rendered inefficient by mutation mechanisms linked to viral escape that lower the expression of the major histocompatibility complex (MHC) or mutate the epitope-target of such cells (Idrees and Sohail 2021). Therefore, chimeric antigen receptor (CAR) $\mathrm{T}$ cells represent an attractive alternative.

However, CAR receptors built on CD4+ T elements have been shown to make CAR-T cells susceptible to HIV infection. To overcome this potentially harmful situation, the quot;CD4CARquot; system was equipped with a viral fusion inhibitor (C46 peptide) or small hairpin RNAs to break down the HIV-1 co-receptor (CCR5) and degrade the RNA viral (Zhen et al. 2017). Both methods made the "CD4CAR-T" system resistant to HIV infection and gave them a long persistence and better control of HIV infection in vivo (Sohail et al. 2021).

In addition, several genome editing techniques have been used to eliminate CCR5 in T cells to give them lifelong resistance to HIV infection. These include the use of various nucleases including ZFN (zinc finger nuclease), which have shown promise in some clinical studies (NCT00842634 and NCT01044654), TALEN (transcription activator-like nuclease), and others nucleases in preclinical studies (Shi et al. 2017). These endonucleases were already used to produce universal CAR-T cells by breaking down TCR (Ren et al. 2017). At present, it is believed that a test would be needed to verify their ability to break down CCR5 in HIV-CAR T cells.

Finally, to overcome the mechanism of HIV escape from the immune response, $\mathrm{T}$ cells expressing bi and tri-specific CARs were designed that target up to three HIV antigens to increase both specificity and affinity.

In this case, the CD4 element was fused with a specific quot;scFv\&quot; complex for a CD4-induced epitope on gp120 or the carbohydrate recognition domain (CRD) of a human C-type lectin that binds to glycans stored on virus envelope (Ghanem et al. 2018). The bi-specific CD4-anti gp120 scFv CAR had better HIV activity than CD4 alone. CARs presenting a CD4-mannose- binding lectin (MBL) showed better activity than both CD4 alone and CD4-anti gp120. However, as type $\mathrm{C}$ lectins can bind glycans that are not specific to HIV-infected cells and can be associated with healthy cells, off-target targets cannot be ruled out and this is an aspect that needs to be analyzed and evaluated. carefully.

The hypothesis developed by our research group is that there may be a positive action in the use of these adoptive immunotherapy systems also against SARS-CoV2 infection, admitting the need for adjustments concerning the epitopetarget (RBD domain of SARS-CoV2 protein S, i.e) and remodeling the action of nucleases within the action of the same virus. The adoption of bi-specific or tri-specific CAR-T could be a solution, even if it is necessary to carefully evaluate the quot;off- targetquot; objectives and, consequently, to re-engineer the construct to limit any collateral damage. 
The rest of the manuscript is organised as follows: In the next section, we will discuss the probability-based approaches for the infected cell and the immune cell cross talk. We will present model and some useful conclusions are drawn afterwards.

\section{Kinetic modeling}

\section{The role of Moran and branching process in viral infection invasion}

To define Moran process, consider a population having constant size (say $M$ ) with two types of individuals (e.g. $A$ and $B$ ), at each time step a random individual (which is of either type $A$ or $B$ ) is chosen to reproduce and same as one is chosen to die so that the population size remains constant, the reproduction rate depends on fitness and both the types can have different fitness. If the fitness of type $A$ is set to 1 and the fitness of type $B$ is set to $r$ then probability that type $A$ is chosen to reproduce and type $B$ is chosen to reproduce are, respectively,

$$
\begin{aligned}
& P(A)=\frac{r i}{r i+(N-i)} \\
& P(B)=\frac{N-i}{r i+(N-i)} .
\end{aligned}
$$

Mathematically, the Galton-Watson process is the most commonly used formula for simulating a branching process. It takes the form:

$Z_{n+1}=\sum_{i=1}^{Z_{n}} X_{n, i}$

with $Z_{0}=1$. In the above equation $Z_{n}$ is the state in the period n, and $X_{n, i}$ denotes the random variables representing the number of direct successors of member $i$ in period $n$, where all random variables have the same probability distribution and all are mutually independent over all $n \in\{0,1,2, \ldots\}$ and $i \epsilon\left\{1, \ldots, Z_{n}\right\}$.

Alternatively, a random walk can also be used to formulate a branching process. The equation is as follows:

$S_{i+1}=S_{i}+X_{i-1}-1=\sum_{j=1}^{i+1} X_{j}+1$,

with $S_{0}=1$. In the previous equation; $S_{i}=$ state in period $i ; X_{i}=$ random variable where all random variables have the same probability distribution and all are mutually independent over all $i$.

Branching processes have been implemented widely in bio-mathematical modeling. Recently, Altrock et al. (2015) provided a detailed literature review highlighting the importance of basic and clinical research on cancer, in which extensive details were documented relating to methodologies for analyzing and explaining cancer at several scales by mathematical modeling.

Viral infections have become as threatening to the world as cancers, and inspired from the studies on the CAR-T therapies of cancer, scientists have now documented useful trial based literature to support the hypothesis of treating SARS-2 infection with CAR-t cells. Such studies requires a comprehensive investigation from the onset. Predictions about the fate of the disease may eventually help to implement successfully some preventive measurements.

For this purpose mathematical models can be very useful. Stochastic modelling is particularly useful since it allows randomness to be incorporated and more accurately reflects actual disease phenomena. Therefore, another approach in branching process simulation is to use a stochastic process which is also based on prediction; this process describes the evolution in a system of random variables with time. In this way stochastic modeling has been contributing substantially and fruitfully to improving our understanding of cancer evolution.

Recently Komarova (2006) framed and scrutinized a stochastic model for the resistance of multi-drug during the treatment of infected cells/ unhealthy cells, by multi-drug therapies.

The infected cell turnover rate, mutation rate and the dependency towards treatment which is known to influence the early tumor load, has also been investigated in the model presented in Komarova (2006), wherein the author describes the general principles of the development and evolution of resistant cells, before and after the start of the treatment. In Komarova (2006), it was also observed that succession of the treatment was independent of the turnover rate for single drugs and was found to be opposite for multi-drug therapies.

The probability of treatment success is directly proportional to the number of drugs used for low turnover rates whereas it is inversely proportional for high turnover rates.

During the progression of the accumulation of SARS-2 infected cells population, the driver and passenger mutations are found (Rochman et al. 2021) and this accrual can also be examined by a discrete time branching process. In this manuscript, a mapping is developed for SARS-2 inspired from cancer mutation; therefore, it worth mentioning the contribution of Bozic et al. (2010), where they presented a model that described the relationship of drivers and passengers mutation in the growth of diseased cells population. Initially, it starts with a single driver mutation and then its clonal expansion accrues more mutations, showing that the death rate is reducing slightly in each mutation and therefore the clonal expansion rate is increased by each new driver mutation. Moreover, in the growth of tumor this model 
provides a relatively simple formulation for the number of driver mutations as a component of the aggregate number of mutations. The actual selective advantage which is given by regular somatic mutation in human tumors is that its location is calculated by this model when applied to the experimental data. Selective advantage though exceptionally small steps proceeds to have great potential for experimental analysis.

\section{Mathematical kinetics and mutation}

It is a fact that there are several types of natural noises in biological systems due to different communications among the cells. This phenomena leads to the fact that such systems are not purely deterministic and involve some randomness, mainly consisting of internal (thermal fluctuations) or external (mainly conformational) noises. Such noises have a vicious part in natural processes and lead system towards abnormalities or sometimes entirely random behavior.

For better perceptive of biological systems it is thus necessary to add stochastic effects in mathematical models of biological and biochemical systems. Kramer's seminal paper (1940) has been a prototype for a thermal activation process continually. Lately, activation in the presence of time varying fields has become a topic of vast significance because of the findings of many counterintuitive noise supported special effects, such as stochastic resonance (Gammaitoni et al. 1998) or movement in Brownian motors (Sherin et al. 2018).

A relevant mesoscopic approach in which the time evolution of a collective coordinate is overseen by stochastic differential equations may be used to study the dynamics of such complex biological systems (Horsthemke 1984).

In such an approach, system kinetics resembles to Kramer's situation with a Brownian particle wandering over an effective free energy potential. The Brownian particle may experience a potential that is influenced by some random oscillations with characteristic time scale which is comparable with one of the time scales governing the passage over the potential barrier separating basins of the stationary points of the biological system. According to the stochastic model, tumor cells are biologically equivalent but their behavior is influenced by intrinsic and extrinsic factors and is therefore both variable and unpredictable. One of the major roles of the immune system is to recognize and eradicate the tumor cells on the basis of their expression of tumor specific-antigens or molecules provoked by cellular stress. Such process is named as tumor immune surveillance in which cancerous and/or precancerous cells are killed by immune system before they can cause harm. Many experiments on mouse model of cancer and human with cancer have provided a convincing evidence about tumor extinction i.e., particular immune cell types, effector molecules, and pathways can sometimes mutually function as extrinsic tumor suppressor mechanism.

Therefore, a detailed theoretical study on the mechanisms of the interaction between virus infected cells and the immune system is necessary for planning efficient strategies of the treatment (Sohail and Nutini 2020; Yu et al. 2020, 2021). An illustration of a coupling between the external noise and a collective variable is an effect of cell-mediated immune surveillance against the cancer (Garay and Lefever 1978).

In biochemistry, 'Michaelis-Menten' kinetics is one of the best-known models of enzyme kinetics. The model takes the form of an equation describing the rate of enzymatic reactions, by relating reaction rate to the concentration of a substrate. A basic model can be defined as follows:

$S+E \underset{k_{-1}}{\stackrel{k_{1}}{\rightleftharpoons}} S \cdot E->\left[k_{2}\right] P+E$,

where $S$ is the substrate, $E$ is the enzyme, $S E$ is the substrateenzyme complex, $P$ is the product, and $k_{1}, k_{-1}$ and $k_{2}$ are constants. Let $s, e, c$ and $p$ be the corresponding concentrations of the substrates $S, E, S E$ and $P$, respectively. According to the Law of Mass Action (LMA) "the rate of a reaction is proportional to the product of the concentration of the reactants". The enzyme reaction illustrated above based on LMA forms the following system of differential equations (see Fig. 1):

$\frac{\mathrm{d} s}{\mathrm{~d} t}=-k_{1} e s+k_{-1} c$,

$\frac{\mathrm{d} e}{\mathrm{~d} t}=-k_{1} e s+\left(k_{-1}+k_{2}\right) c$,

$\frac{\mathrm{d} c}{\mathrm{~d} t}=k_{1} e s-\left(k_{-1}+k_{2}\right) c$

$\frac{\mathrm{d} p}{\mathrm{~d} t}=k_{2} c$,

$s(0)=s_{0}$

$e(0)=e_{0}$

$c(0)=c_{0}$

$p(0)=p_{0}$,

where $k_{1}, k_{-1}, k_{2}, s_{0}$ and $e_{0}$ are positive constants (see Table 1).

The cell-mediated immune surveillance against SARS-2 is one of the scientific topics under discussion these days and it may be described in terms of a "predator-prey" system 
Table 1 Description of variables

\begin{tabular}{ll}
\hline Variable & Description \\
\hline$s(t)$ & Virus infected cells \\
$e(t)$ & CTL and CAR-T action \\
$c(t)$ & Complex \\
$p(t)$ & Dead cell count w.r.t. time. \\
\hline
\end{tabular}

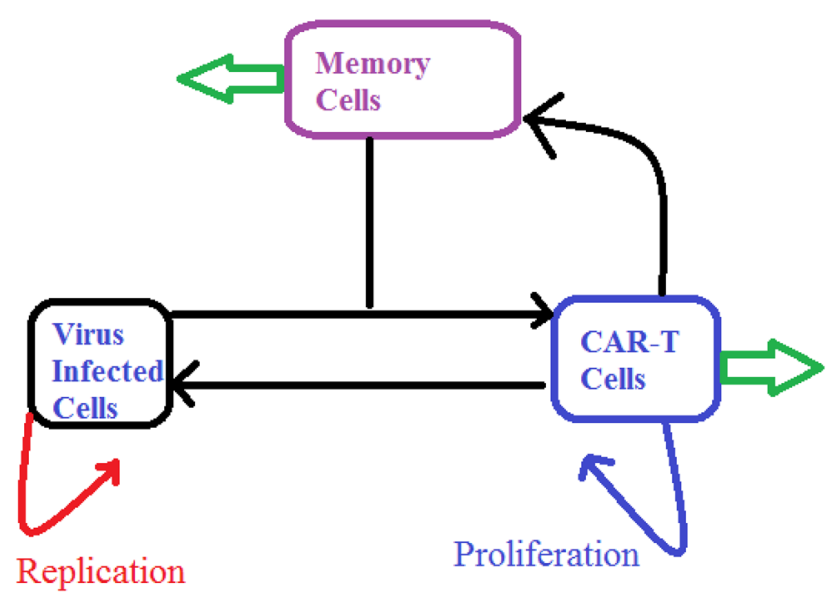

Fig. 1 CAR-T cells, memory cells and the infected cells

and be approximated by a saturating, enzymatic like process whose time evolution equations are similar to the standard Michaelis-Menten kinetics. The population of SARS-2 infected cells plays the role of "preys" and immune cells act as "predators".

The population dynamics can be defined as follows: First, the cytotoxic cells bind to the virus infected cells at a rate proportional to the kinetic constant $k_{1}$; second, the virus infected cells which have been found are killed and the complex dissociates at a rate proportional to $k_{2}$. The process can be illustrated as

$X+Y \stackrel{k_{1}}{\rightarrow} Z \stackrel{k_{2}}{\rightarrow} Y+P$

where $X, Y, Z$ and $P$ represent the population of virus infected cells, active cytotoxic cells as well as the CAR-T cells, bound cells and the dead cells, respectively (OchabMarcinek and Gudowska-Nowak 2004) (see Fig. 2).

A lot of work has been done to analyze the effect of noise in the disease dynamics. Marcinek et al. (2004) discussed properties of two state cancer growth model subject to independent Markovian dichotomous noise (affecting the immunological response) and to an additive thermal noise, by considering an overdamped Brownian particle moving in a two well quasi potential between reflecting and absorbing boundaries. Random fluctuations of the environmental conditions may disturb the enzyme activities which in return may deviate to rate of formation from the mean value in the biochemical reactions. The role of such fluctuations might be critical for whole system. Many environmental factors like, the supply of oxygen, the supply of nutrients, chemical agents, gene expression, degree of vascularization of tissues, the immunological state of the host, temperature, radiations, protein synthesis and antigens influence viral infection. As a consequence of this, the system faces some inescapable stochastic effects.

Marcinek et al considered a system in which the modulating activity of the immune response is modeled as a dichotomous random process of the relative rate of neoplastic cell destruction. They discussed physical aspects of environmental noises in such a system and pointed out the chance of co-occurrence of dynamical systems where noise enhanced stability (NES) and resonant activation (RA) phenomena may occur together, such mechanism was explained by examining the variance of first passage times as a function of noise intensity. Moreover, in Fiasconaro et al. (2008), Fiasconaro et al. explored the dependence between the intensity of external fluctuations and the transition time from the state of a stable tumor and to the state of its extinction under the influence of a moderate periodic treatment and understood a competition between the probability of extinction and the reappearance of cancer cells in the system to analyze optimal conditions of its control. Marcinek found that random dichotomous fluctuations in the immune response intensity along with Gaussian environmental noise lead to emergence of a spatial pattern of two phases, in which cancer cells, or, respectively, immune cells predominate (Ochab-Marcinek and Gudowska-Nowak 2004).

\section{Results and discussion}

In this manuscript, the CAR-T cells action against the SARS-2 infected cells is discussed. Some mapping are developed, including the Moran process and the kinetic modeling. We have used the Matlab algorithm to generate the two different trajectories for the Moran type process which gives the number of $\mathrm{N}$ type proteins in a population of cells. The two different trajectories subject to different dynamical interaction between the CAR-T cells and the antigen are presented in Fig. 3.

Next, in Figs. 4 and 5, we have presented the dynamics relative to the change in infected cells count, relative to the three rates of the two coefficients for the model (Eqs. 6-9). We can see that the amplitude decreases but the perturbation increases possibly due to the action of multiple associated factors such as the changes in epitope expression, complex formation rates and the death rates. 

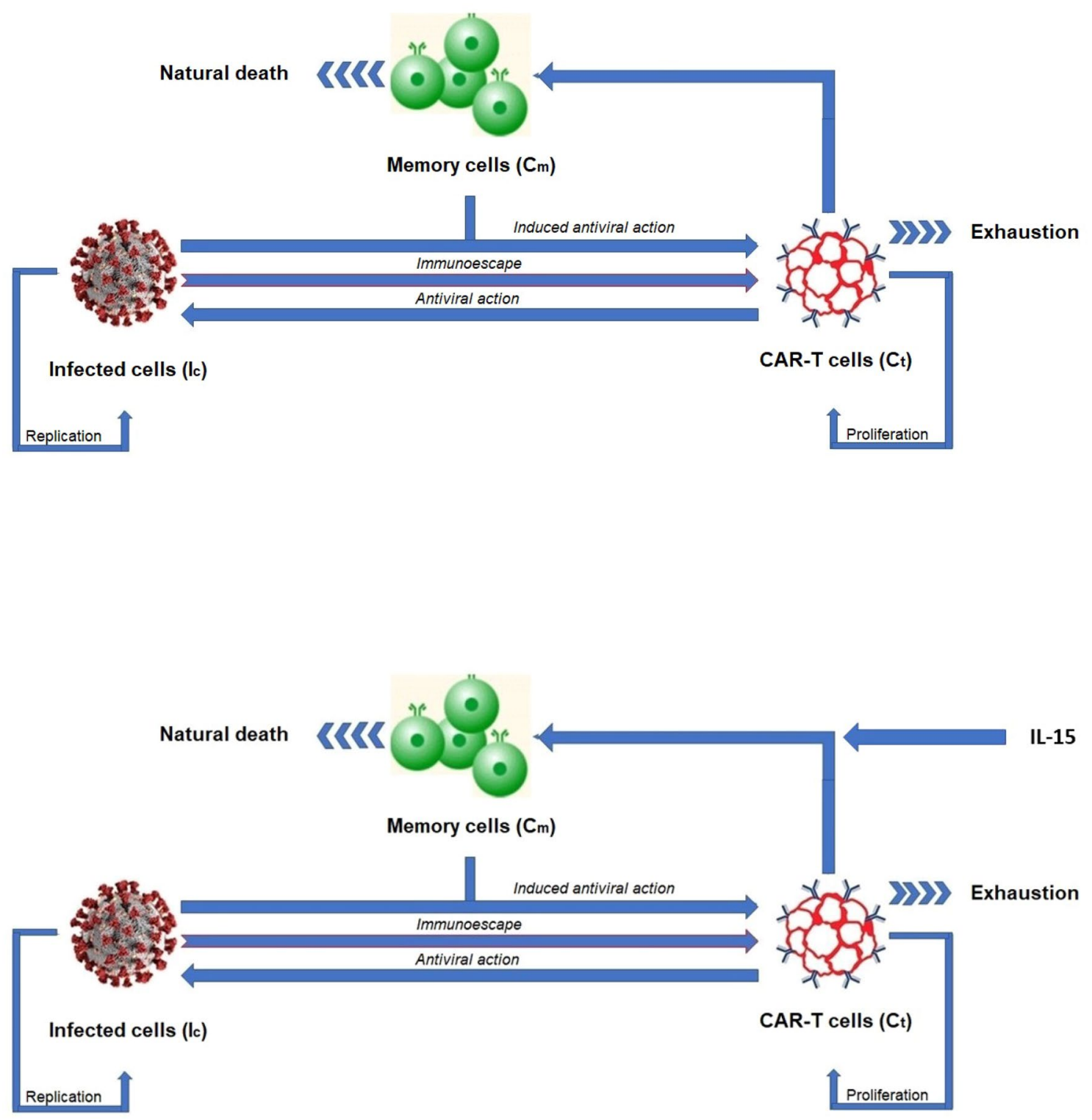

Fig. 2 Schematic depiction of the CAR-T cells and antiviral action against SARS-CoV2

Next in Fig. 6, we can see the dynamics of the substrate, enzyme, complex and the dead cells densities relative to two population sizes of the injected CAR-T cells, left $(N=$ $5000)$, right $(N=10,000)$. We can see the dispersion and the stochastic perturbation in the interaction dynamics.

\section{Conclusions}

The model represented here is based on the hypothesis that the antiviral therapy against SARS-CoV2 is possible through CAR-T cells. The viral target constituted by the $\mathrm{N}$ protein ( typical of this viral species) is discussed.
Although some of these assumptions are not currently experimentally verifiable parameters, they appear to be deeply interconnected according to a logic based on proven experimental events such as memory induced by CAR-T therapy or the presence of CAR exhaustion. -T cells themselves (Kasakovski et al. 2018). It is evident that there is a viral immuno-escape that remains contained thanks to the action of CAR-T cells. Furthermore, elements of analysis conducted using a MM model, evaluates the impact of CAR-T cells obtained with the use of Treg cells in viral infection, considering both the exhausted condition and the action of the IFN-cytokine in therapy. The quantitative results validate the "CAR-T and SARS-CoV2 hypothesis". The model can be extended further and can show efficiency 
Fig. 3 Moran process for the $\mathrm{N}$ protein bindings

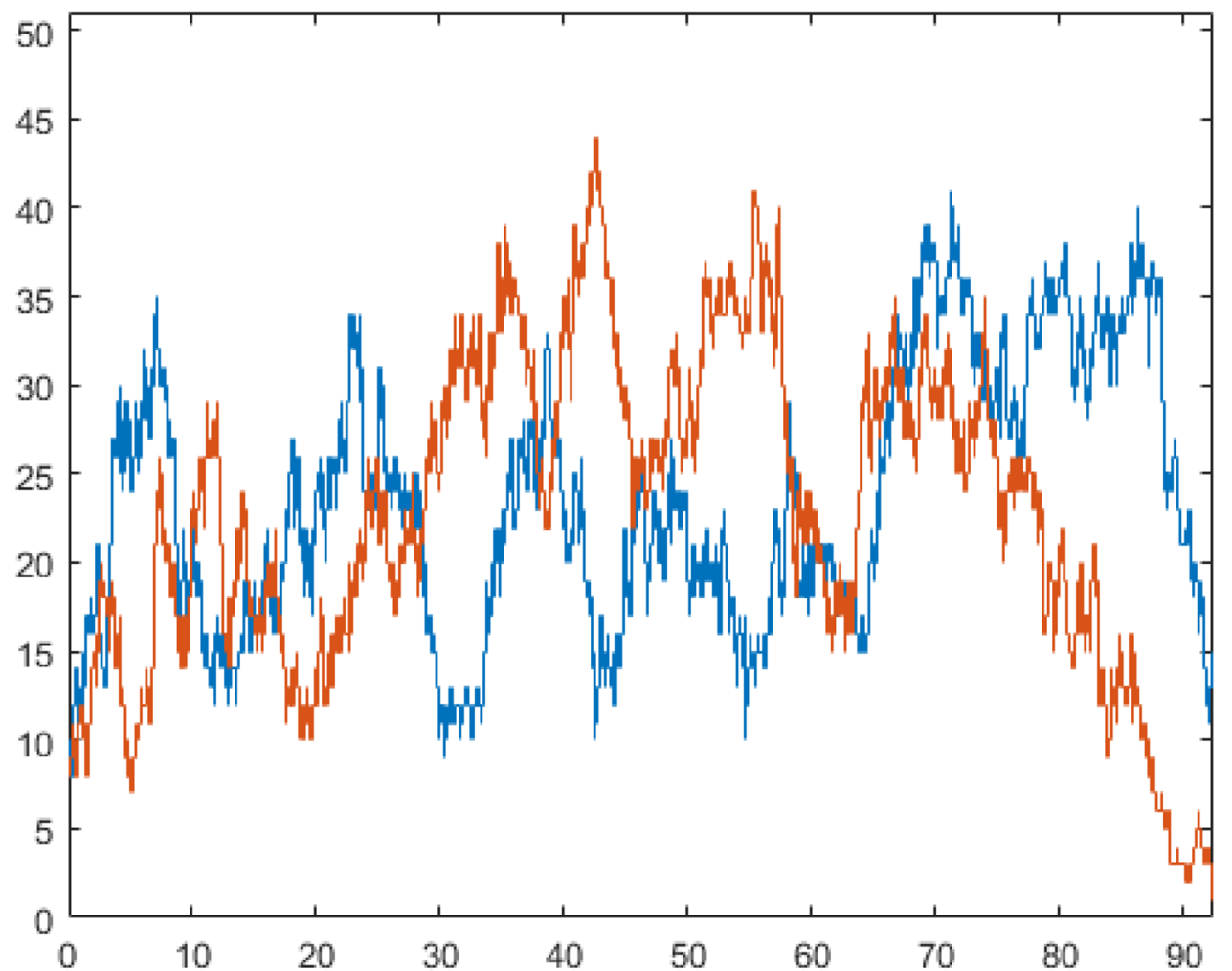

Fig. 4 Kinetic rate variations for the infection control

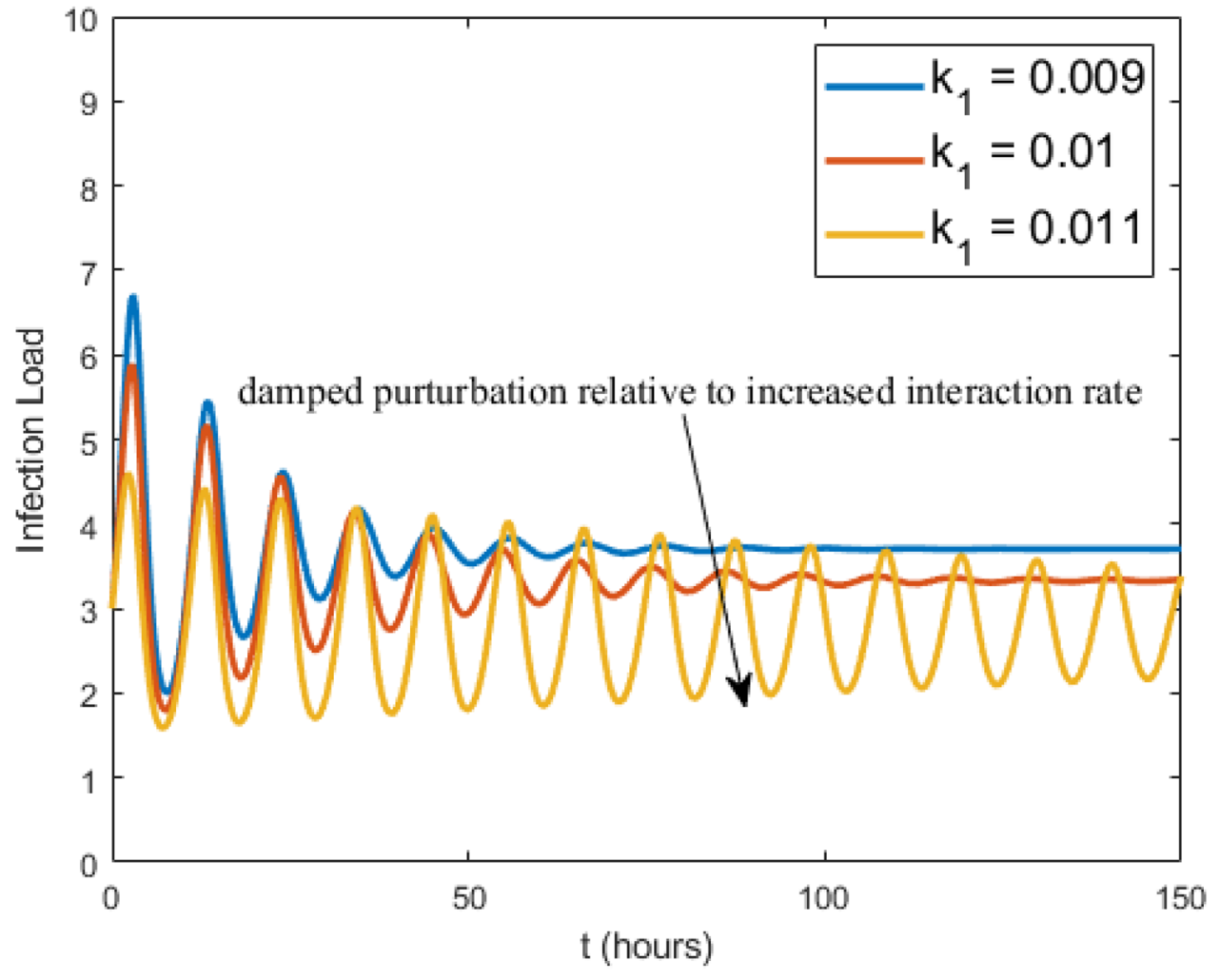

of the therapy in the case of severe COVID-19 infection. Although the extent of the side effects of this type of therapy has to be assessed, the computational analysis shows a possible efficacy of CAR-T cells in containing the COVID19 syndrome and is, therefore, a good rational basis for further investigation. 
Fig. 5 Kinetic rate variations for the infection control

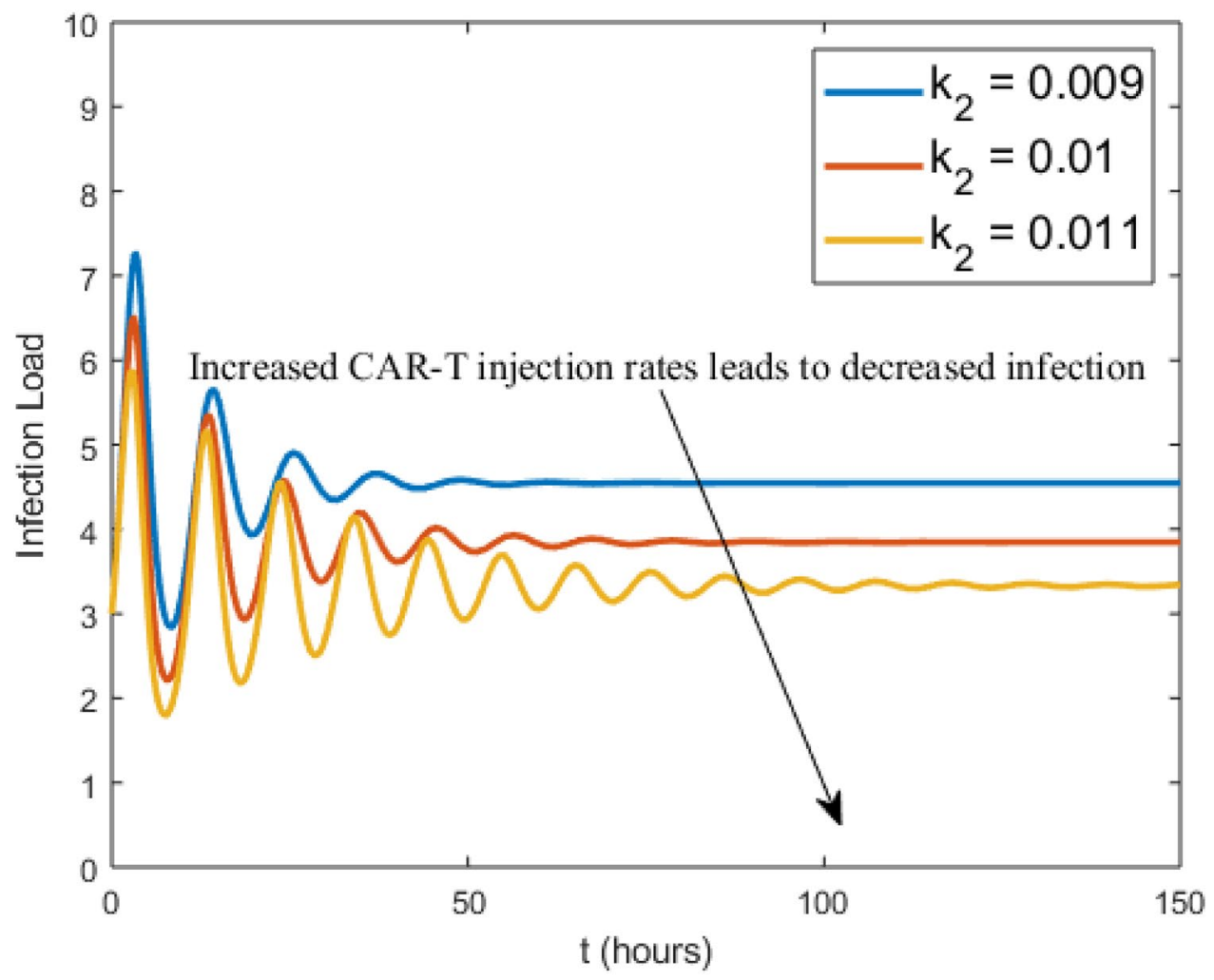

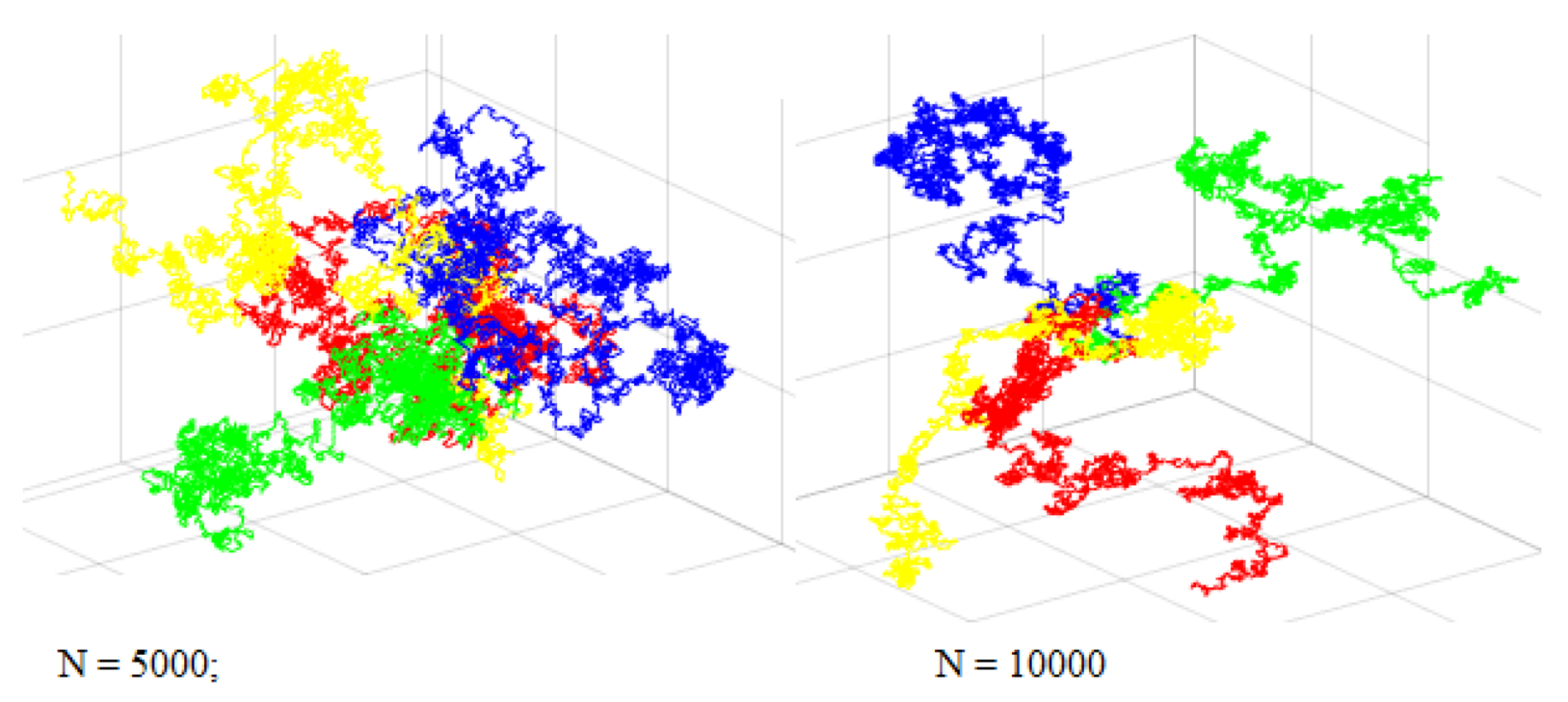

Fig. 6 Stochastic process for substrate enzyme model

The model quantifies a rather incisive therapeutic action and is able to better quantify some assumptions:

- The CAR-T cells, obtained from Treg cells, optimal sub-population as suggested in the literature (VeigaParga et al. 2013; Che et al. 2015), are activated and directed against the target, obtaining a cytotoxic effect.
- CAR-T cells obtained from the sub-population of Treg cells are also able to express a containment of an excessive immune response, regulating its effects and staying longer in the organism.

- The CAR-T cells have an inactivation rate (exhaustion).

- CAR-T cells kill infected cells at a constant rate. 
- The viral infection performs an action of "escape" from the immune system function (in the case of SARS-CoV2 this action is carried out by the "novelty" represented by the virus itself towards the immune system).

- A part of the CAR-T cells constitute immunological memory.

- The proliferation of CAR-T cells is constant.

\section{References}

Altrock PM, Liu LL, Michor F (2015) The mathematics of cancer: integrating quantitative models. Nat Rev Cancer 15(12):730-745

Bozic I, Antal T, Ohtsuki H, Carter H, Kim D, Chen S, Karchin R, Kinzler KW, Vogelstein B, Nowak MA (2010) Accumulation of driver and passenger mutations during tumor progression. Proc Natl Acad Sci 107(43):18545-18550

Che JW, Kraft AR, Selin LK, Welsh RM (2015) Regulatory t cells resist virus infection-induced apoptosis. J Virol 89(4):2112-2120

Cheng Y, Wong MT, van der Maaten L, Newell EW (2016) Categorical analysis of human $t$ cell heterogeneity with one-dimensional soli-expression by nonlinear stochastic embedding. J Immunol 196(2):924-932

Elinav E, Waks T, Eshhar Z (2008) Redirection of regulatory t cells with predetermined specificity for the treatment of experimental colitis in mice. Gastroenterology 134(7):2014-2024

Fiasconaro A, Ochab-Marcinek A, Spagnolo B, Gudowska-Nowak E (2008) Monitoring noise-resonant effects in cancer growth influenced by external fluctuations and periodic treatment. Eur Phys J B 65(3):435-442

Gammaitoni L, Hänggi P, Jung P, Marchesoni F (1998) Stochastic resonance. Rev Mod Phys 70(1):223

Garay RP, Lefever R (1978) A kinetic approach to the immunology of cancer: stationary states properties of efffector-target cell reactions. J Theor Biol 73(3):417-438

Ghanem MH, Bolivar-Wagers S, Dey B, Hajduczki A, VargasInchaustegui DA, Danielson DT, Bundoc V, Liu L, Berger EA (2018) Bispecific chimeric antigen receptors targeting the cd4 binding site and high-mannose glycans of gp120 optimized for anti-human immunodeficiency virus potency and breadth with minimal immunogenicity. Cytotherapy 20(3):407-419

Horsthemke W (1984) Noise induced transitions. In: Non-Equilibrium dynamics in chemical systems. Springer, pp 150-160

Idrees M, Sohail A (2021) Bio-algorithms for the modeling and simulation of cancer cells and the immune response. Bio-Algorithms Med-Syst 17(1):55-63

Kasakovski D, Xu L, Li Y (2018) T cell senescence and car-t cell exhaustion in hematological malignancies. J Hematol Oncol 11(1):1-9

Komarova N (2006) Stochastic modeling of drug resistance in cancer. J Theor Biol 239(3):351-366
Kramers HA (1940) Brownian motion in a field of force and the diffusion model of chemical reactions. Physica 7(4):284-304

Leibman RS, Richardson MW, Ellebrecht CT, Maldini CR, Glover JA, Secreto AJ, Kulikovskaya I, Lacey SF, Akkina SR, Yi Y et al (2017) Supraphysiologic control over hiv-1 replication mediated by cd8 $\mathrm{t}$ cells expressing a re-engineered cd4-based chimeric antigen receptor. PLoS Pathog 13(10):e1006613

Ochab-Marcinek A, Gudowska-Nowak E (2004) Population growth and control in stochastic models of cancer development. Phys A 343:557-572

Ren J, Zhang X, Liu X, Fang C, Jiang S, June CH, Zhao Y (2017) A versatile system for rapid multiplex genome-edited car $t$ cell generation. Oncotarget 8(10):17002

Roberts MR, Qin L, Zhang D, Smith DH, Tran A-C, Dull TJ, Groopman JE, Capon DJ, Byrn RA, Finer MH (1994) Targeting of human immunodeficiency virus-infected cells by CD8+ T lymphocytes armed with universal T-cell receptors (1994):2878-2889

Rochman ND, Wolf YI, Faure G, Mutz P, Zhang F, Koonin EV (2021) Ongoing global and regional adaptive evolution of sars-cov-2. Proc Natl Acad Sci 118(29)

Scholler J, Brady TL, Binder-Scholl G, Hwang W-T, Plesa G, Hege KM, Vogel AN, Kalos M, Riley JL, Deeks SG et al (2012) Decadelong safety and function of retroviral-modified chimeric antigen receptor t cells. Sci Transl Med 4(132):132ra53-132ra53:132ra53

Sherin L, Farwa S, Sohail A, Li Z, Bég OA (2018) Cancer drug therapy and stochastic modeling of "nano-motors". Int J Nanomed 13:6429

Shi B, Li J, Shi X, Jia W, Wen Y, Hu X, Zhuang F, Xi J, Zhang L (2017) Talen-mediated knockout of ccr5 confers protection against infection of human immunodeficiency virus. JAIDS J Acquir Immune Defic Syndr 74(2):229-241

Sohail A, Nutini A (2020) Forecasting the timeframe of coronavirus and human cells interaction with reverse engineering. Progress Biophys Mol Biol 155:29-35

Sohail A, Tunc S, Nutini A, Arif R (2021) Furin and the adaptive mutation of sars-cov2: a computational framework. Model Earth Syst Environ, Springer, pp 1-10

Veiga-Parga T, Sehrawat S, Rouse BT (2013) Role of regulatory t cells during virus infection. Immunol Rev 255(1):182-196

Yu Z, Sohail A, Nutini A, Arif R (2020) Delayed modeling approach to forecast the periodic behaviour of sars-2. Front Mol Biosci 7:386

Yu Z, Ellahi R, Nutini A, Sohail A, Sait SM (2021) Modeling and simulations of covid-19 molecular mechanism induced by cytokines storm during sars-cov2 infection. J Mol Liq 327:114863

Zhen A, Peterson CW, Carrillo MA, Reddy SS, Youn CS, Lam BB, Chang NY, Martin HA, Rick JW, Kim J, Neel NC (2017) Longterm persistence and function of hematopoietic stem cell-derived chimeric antigen receptor $\mathrm{T}$ cells in a nonhuman primate model of HIV/AIDS. PLoS Pathog 13(12):e1006753

Publisher's Note Springer Nature remains neutral with regard to jurisdictional claims in published maps and institutional affiliations. 